varied $^{5} 6$ between $38 \%$ and $81 \%$. Many of the women who present for "screening" already have symptoms, and no doubt factors such as these contribute to the wide variation in the cancer detection rates in different studies. Representative figures range from 2.7 per 1000 (aged 40-69 years) at the initial screening examination in the HIP trial, ${ }^{7}$ to 7.6 per 1000 (aged 35-75 years, self-selected), ${ }^{8}$ to 11.3 per 1000 (aged 35 years or more, "high risk") 9 and $12 \cdot 0$ per 1000 (aged 40 years or more, self-selected). ${ }^{10}$ Even higher rates have been reported $^{8}$ (24.5 per 1000 "high risk, well women").

A number of different screening methods are under investigation, but most reports are based on the combination of clinical examination and mammography. The HIP data suggest that both these modalities are essential, since $44 \%$ of cancers would have been found only by the former and $34 \%$ only by the latter. Most other authors agree with the conclusions reached by the New York group. However, mammography is less effective in premenopausal women, in whom the breasts are homogeneously dense. Since it is this group of women that may be at risk of a radiation-induced breast malignancy, it is probably best to restrict mammography as far as possible to the over-40s. ${ }^{11}$

Another unresolved problem is the optimal frequency of screening. In the HIP trial $15 \%$ of all cancers diagnosed were detected within a year of a normal screening examination, ${ }^{4}$ while in the Guttman Institute study ${ }^{4}$ the corresponding figure was $9 \%$. These data are difficult to interpret; some of the "interim" tumours must represent the result of "falsenegative" screening, while others presumably arose de novo between examinations.

To co-ordinate British research the DHSS and the MRC have established working parties to study the service and biomedical aspects of a national screening programme. Four centres (Bath, Ealing, Edinburgh, and Manchester) are concerned at present, and some results from Ealing were published last year. ${ }^{10}$ The paper at page 858 is a report from the screening clinic in the University Hospital of South Manchester. Using clinical examination and mammography the non-medical team of nurse and radiographer was as successful at detecting breast abnormalities as a team of surgeon and radiologist. The overall cancer pick-up rate was 8.4 per 1000 , or 4.6 per 1000 in asymptomatic women. Four methods of mammography were used, and it was found possible to obtain satisfactory plates with a mean upper inner quadrant dose as low as $0 \cdot 1-0.5 \mathrm{rad}$. The response rate of those invited for screening was $54 \%$ and was highest in the youngest women. Social class did not affect this response rate, but those who referred themselves were predominantly from the upper social classes. Among the women invited for tests the biopsy rate was $2.9 \%$ (other studies ${ }^{4} 10$ have reported rates from $2.4 \%$ to $9 \cdot 8 \%$ ). Extrapolation of these findings to all women aged 40-65 years in Manchester would imply that a full screening programme would lead to surgeons carrying out an extra 20 biopsies per week.

Many people believe that eventually any screening programme should be universally available, so that careful consideration has to be given to economic factors. In 1971 it was calculated that the use of mammography to screen all women aged 40 years and over in the USA would cost $\$ 622$ million annually. ${ }^{12}$ Knox $^{13}$ estimates that at 1975 prices such a scheme would cost not more than $£ 12$ million annually in England and Wales. His simulation model indicated that the programme would produce a $12 \%$ reduction in mortality from breast cancer on the assumption of a $50 \%$ uptake and 30 successive annual screening examinations; thus the cost per "life saved" would be $£ 8000$. However, $95 \%$ of women will never develop breast cancer, so much discussion has centred on the definition of high risk groups for selective screening. Unfortunately the known correlates of the disease appear to have little predictive power, ${ }^{14}$ but Knox has calculated that a selective programme aimed at a $10 \%$ reduction in mortality might be achieved at a cost of $£ 2000-£ 3000$ per "life saved."

There are other constraints on breast cancer screening. They include the availability of personnel and equipment, but the results of the Manchester study suggest that these problems are not insoluble.

${ }^{1}$ Armstrong, B, International fournal of Cancer, 1976, 17, 204.

2 Fisher, B, et al, New England fournal of Medicine, 1975, 292, 117.

3 Bonadonna, G, et al, New England fournal of Medicine. 1976, 294, 405.

4 Strax, P, Fournal of the American Medical Association, 1976, 235, 1600.

${ }^{5}$ Barnes, S, et al, Lancet, 1968, 1, 1417.

${ }^{6}$ Langeland, P, Acta Radiologica, 1970, suppl. No 297.

Shapiro, S, et al, in Seventh National Cancer Conference Proceedings, p 663. Lippincott, Philadelphia, 1973.

${ }^{8}$ Stark, A M, Lancet, 1976, 1, 359.

9 Thomas, B A, Lancet, 1975, 2, 914

${ }^{10}$ Chamberlain, J, et al, Lancet, 1975, 2, 1026

11 Mole, R H, unpublished information, 1976.

12 Dowdy, A H, et al, Cancer, 1971, 28, 1558.

${ }^{13} \mathrm{Knox}, \mathrm{E} \mathrm{G}$, in Probes for Health: Essays from Health Services Research Centre, p 38. Oxford University Press, Birmingham, 1975.

${ }^{14}$ Shapiro, S, et al, in Host-Environment Interactions in the Etiology of Cancer in Man, p 169. International Agency for Research on Cancer, Lyon, 1973,

\section{Safety for children}

Accidents are the leading cause of death in childhood, and one in six of all admissions of children to hospital are the result of trauma. Yet recent attempts to evaluate the official returns of accidents in childhood have shown the information so inadequate and misleading that little reliance can be placed upon it. Nowhere is this more apparent than with the leading causes, road traffic and the home.

In the case of road accidents the returns (based on information collected by the police at the scene of the accident) of serious and slight injuries rely on definitions which bear little relation to clinical severity or to prospect of permanent disability. No distinction is made between a broken finger and paraplegia. There is considerable under-reporting and misreporting within this unsatisfactory classification, with an uneven distribution of errors between different categories of road user. The quality of the data is worst for cyclists and pedestrians, who are most exposed to serious injury. Only those child cyclists in collision with motor vehicles are officially reported as injured-but they represent only $11 \%$ of the total. Similarly, the Registrar General's returns of mortality show that over half of the fatal domestic accidents are among the over $65 \mathrm{~s}$, and this finding is generally assumed to reflect the age distribution of accidents. However, a recent survey ${ }^{1}$ of one year's admissions to accident and emergency departments in six different areas of England showed that only 13\% of home accidents were in the over $65 \mathrm{~s}$ and that $30 \%$ were in children aged under 5 .

It was against this background of inadequate information that last month an international conference, sponsored by the Medical Commission on Accident Prevention and the Department of Child Health of the University of Newcastle on Tyne, discussed the theme of "children, the environment, and accidents." To what extent should the environment be adapted to make it safer for children? Should children be protected 
from all the risks of their natural environment? Is it necessary to take all the fun out of play ? Such questions help us to place in perspective the considerable economic cost of major modifications to the environment.

The ultimate recipe for pedestrian safety is total segregation of pedestrians from traffic, and successful attempts in this direction have been made in new towns such as Stevenage. But what happens when children protected in this way are eventually exposed to traffic? The solution to the problem must lie in creating an environment in which the child can adapt gradually to its surroundings through progressive stages of its development. It is the unnecessary hazards that should be removed-and modern domestic architecture is a chief offender. Architects frequently fail to appreciate dangers to children, and when rules have been drawn up to avoid dangers they are widely ignored. Furthermore, devices such as safety window catches last for only a fraction of the expected life of the building. Dr W R Sinnott, from Warley College of Technology, told the conference that he had had no difficulty in collecting examples of the highly dangerous aspects of much modern domestic architecture: he had simply made slides of some of the prize winning designs illustrated in our architectural journals. Official advice seems of little help here. One booklet issued by the Department of the Environment recommends a design incorporating features expressly identified as dangerous to children in another booklet issued by the same department.

Road traffic is the child's most complex and most challenging environment, and it is not surprising that road accidents are the chief cause of serious injury to children. Some $80 \%$ of all recorded injuries to children in road accidents occur while the child is crossing the road, and a quarter occur in the road where the child lives. Parents generally overestimate the ability of their children to cope. A recent survey ${ }^{2}$ showed that $19 \%$ of mothers with children aged 2 years considered it safe for their children to cross what they admitted to be a very busy road without supervision. The behaviour of adults themselves in crossing roads often sets a deplorable example to children. One-third of the preschool children injured crossing the road are in the charge of an adult at the time of the accident. In another recent study in Hampshire ${ }^{3}$ four out of five children were running across the road when hit, and three out of five did not look before crossing. Only $11 \%$ of children both looked before crossing and saw the vehicle which hit them.

Britain has the worst child pedestrian casualty rate in western Europe ${ }^{4}$ so that it might have been expected that our primary schools (which include the age group at highest risk) would pay close attention to prevention. In practice, only $38 \%$ of primary schools in England and Wales have a planned system of road safety instruction in their curricula, and very little information is available to the teachers concerned about the most important aspects of child pedestrian safety. Last month the Department of the Environment ${ }^{5}$ announced a five-week campaign costing $£ 880000$ to reduce child casualties on the road, timed to coincide with return to school. The campaign includes motivational posters warning motorists to watch out for children, though it is known that such messages are largely ineffective. Why does the Government still put its faith in propaganda rather than in effective legislation? Britain is almost alone in Europe in allowing pedestrians to cross the road when and where they like.

Why, indeed, has so little been done in Britain about childhood accidents ? Perhaps, as Professor Donald Court suggested when summing up at the end of the conference, there is something inevitable and unpredictable inherent in the term "accident" which discourages effective action being taken. Only epidemiological studies can disprove this misconception, and far too few have been carried out. Nor is there much prospect of them being carried out so long as the Government bodies with control of funds for research such as the Transport and Road Research Laboratory obstinately refuse to consider a public health approach to the problem. Meanwhile, there is little evidence of effective liaison in accident prevention between the five Government departments and some 300 national organisations concerned with child welfare. There was therefore considerable support at the conference for the setting up of a national joint committee on accident prevention in childhood, such as has existed for many years in Sweden, where it has achieved impressive results.

\footnotetext{
${ }^{1}$ Department of Prices and Consumer Protection, Collection of Information on Accidents in the Home. London, HMSO, 1976.

Sadler, J, Children and Road Safety: A Survey Amongst Mothers. London, HMSO, 1972.

${ }^{3}$ Grayson, G B, The Hampshire Child Pedestrian Accident Study. Crowthorne TRRL Laboratory Report 668, 1975.

${ }^{4}$ European Conference of Ministers of Transport, Report on Recent Trends in Road Accidents. Paris, 1972.

${ }^{5}$ Department of the Environment, Press Notice No 784, 1 September, 1976.
}

\section{Washing away at acne}

Compelled to brood on any flaw, imaginary or real, in their own state of health the obsessional are unable to relax. Convinced that they must do something to overcome any "disease" that besets them, they fill their medicine cupboards with pills and mixtures and ointments, they purge themselves, they submit to colonic lavage and strange diets and psychoanalysis, and they may traipse from doctor to doctor and clinic to clinic, perpetually unsatisfied by the cool way in which they feel that their problems are viewed. They can be guaranteed to take themselves seriously: and when they get acne they set to work with a will.

The yardstick that the obsessional uses for his comparison is perfection, which in the case of the skin means that it must be totally immaculate-what is commonly called "that schoolgirl complexion," though if the introducer of that term had had a dermatologist's familiarity with the complexion of schoolgirls he might have had second thoughts. There is a general theory, regarded as axiomatic by the obsessional, that skin diseases are due to dirt-a belief fortified by the greasiness and the blackheads present in acne. In addition, acne is regarded as an outward sign of moral defilement, the telltale mark left by all those unhealthy sexual thoughts that well up into the minds of adolescents (and without which we might think that they could scarcely be called adolescent). The inevitable consequence of the obsessional attitude of mind faced with acne is to wash. Washing cleanses physically and morally. The act of washing is also a ritual of purification.

Unfortunately, contrary to the simple rationale based on this assumption, acne is a complicated disease; nor is washing entirely straightforward, for it may have the unexpected effect of aggravating acne, an effect that is magnified and becomes manifest if the washing is done obsessionally. Mills and Kligman ${ }^{1}$ have described this effect and called it acne detergicans, claiming that the harm results from the effect of certain soaps and shampoos in actually producing comedones. It came to light when they saw first one young man and then another who washed themselves vigorously, up to eight times 